\title{
Design of Real-time Safety Accident Prevention Solution for Socially Vulnerable using Object Recognition and Tracking Technology
}

\author{
Sung-Jin Jeoung, Bong-Hyun Kim
}

\begin{abstract}
All countries around the world want to create a safe society and a safe nation. However, crime targeting the socially vulnerable groups continues to increase. This is because the vulnerable groups have physical and physical weaknesses. Therefore, in order to pursue a safe society, it is necessary to protect the socially vulnerable. In this paper, we utilize the latest trend, drones, to protect against vulnerable groups. That is, we designed a real-time accident prevention solution for the elderly, the disabled, children and women using the drones. Drones' autonomous flight technology, obstacle detection technology, collision avoidance technology, wireless communication linkage technology, and real time image transmission technology. Through this, we have studied to enable safe activities in the blind spot of CCTV. In this paper, we have identified the problem of crime prevention system using existing CCTV system. To solve this problem, we have developed a safety accident prevention solution using drones. To this end, we designed the drones to enable real-time tracking by applying autonomous flight technology, obstacle avoidance technique, and object tracking technology. In addition, wireless communication between the object and the drone was applied to prevent flight deviation. And, it is designed to link emergency notification service through real-time video transmission during flight.
\end{abstract}

Keywords : Safety prevention system, Real-time safety, Drone, Obstacle avoidance, Object tracking, Object recognition.

\section{INTRODUCTION}

$\mathrm{O}_{\text {nly one out of every } 10 \text { Koreans feels that our society is }}$ safe. More than half of the women feel afraid of nighttime activities. The survey results show that the number of crimes in Korea is decreasing year by year, but the incidence of sex crimes is steadily increasing. Major crimes such as sexual crimes occur more than $64 \%$ from $6 \mathrm{pm}$ to $6 \mathrm{am}$. Currently, CCTV is used mostly as a safety system. In addition, the monitoring system for hazardous area, security area, and vulnerable area is applied by installing high-quality CCTV for safe facility monitoring[1].

However, the CCTV surveillance system has the inconvenience that it has to be monitored by a staff of 24 hours in the central control and management room. In addition, there are problems such as additional installation and additional financial expenses such as additional

Revised Manuscript Received on July 22, 2019.

* Correspondence Author

Sung-Jin Jeoung, Seoul Business School, Seoul School of Integrated Science \& Technologies, Seoul, Korea. Email: alvinoni87@ gmail.com

Bong-Hyun Kim*, Dept. of Smart IT, U1 University, Yeongdong-gun, Korea.Email: bhkim@u1.ac.kr installation of CCTV due to blind spot generation. In addition to these problems, existing CCTV surveillance solutions have many problems. In particular, it causes many problems such as the occurrence of blind spots, the damage caused by post-damage treatment, the problem of additional installation cost, constitutional portrait rights, confidentiality and freedom of privacy, and infringement of basic rights such as the right to determine self-information. CCTV has changed the unmanned mechanization system of manpower-dependent expense and surveillance system. Some control centers have management personnel, but they are used simply to protect facilities with assets[2,3].

Now, however, it is time to develop solutions for the cost of all facilities that are worth protecting, such as public institutions, homes, and public transportation. Recently, interest in smart cities and smart countries is growing. With the advancement of technology and convenient life, I am interested in "safety" and have a goal of smart age. Therefore, in this paper, we applied the 4th industrial revolution technologies and studied the real - time security preservation solution for the socially vulnerable. To this end, a real-time safety accident prevention solution was designed by using autonomous flight drone, object tracking technology, video transmission technology, and sensor integration integrated module application technology.

\section{SAFETY SYSTEM STATUS}

Socially vulnerable groups refer to the elderly, the disabled, and children. Recently, as sex offenses have increased, interest in safety services has also increased for women. In recent years, social safety net services for protecting vulnerable groups from various incidents and accidents have been strengthened. In other words, it is an institutional device for protecting the people from social risks. In particular, there is a need for greater protection for vulnerable groups.

First, Korea has already entered an aging society in 2000. As of 2017 , the total population is $51,446,000$, of whom $7,076,000$ are aged 65 or older, accounting for $13.8 \%$ of the total population. In 2060 , the elderly population is expected to increase to $41.0 \%$. By age group, the proportion of elderly people aged 80 and older is expected to increase steadily, while those aged 65 to 69 and aged 70 to 79 are decreasing. 


\section{Design of Real-time Safety Accident Prevention Solution for Socially Vulnerable using Object Recognition and Tracking Technology}

These elderly people are part of the socially vulnerable class, and the crimes against elderly people are increasing. According to the number of reports of elder abuse in 2016, $3,093(72.3 \%)$ were elderly women and 1,187 (27.7\%) were male elderly. There was a lot of damage mainly in the large and the $80 \mathrm{~s}$. According to the type of abuse according to gender, both male elderly and female elderly showed high level of emotional abuse and common abuse rate in the order of emotional - physical - abuse. There were 2,078 cases $(41.2 \%)$ of emotional abuse among the elderly women, 652 cases (36.9\%), 462 cases of physical abuse (26.2\%) and 253 cases of abuse (14.3\%, Physical abuse 1,669 cases $(33.1 \%)$ and abuse of abuse 525 cases $(10.4 \%)$ [4].

To the next, crime against persons with disabilities is also steadily increasing. In particular, the victims of sex crimes against persons with disabilities accounted for $47.2 \%$ of all crimes against persons with disabilities. In addition, violence, property extortion, abuse, abuse and other crimes are occurring against persons with disabilities. Recently, crimes targeting people with mental disabilities are increasing. Newly disabled people are less likely to be perpetrators of crime than ordinary people, and are more likely to be at risk of becoming victims of crime. In particular, people with mental disabilities who are admitted to mental health facilities and disabled facilities are at high risk of being exposed to violence and abuse in an environment that is blocked from the outside. Korea's social welfare spending is among the lowest among the 34 OECD countries, and the social integration index, which indicates the degree of social integration, also fell to 21st place in 1995 and 24th place in 2009 compared with OECD member countries. In particular, the social integration index, which includes the number of laws related to the disabled workers and the tolerance of others, is the lowest in the tolerance society segment. Korea is the 14th largest economy in the world, but unemployment and economic polarization are intensifying. On the other hand, in the reality that the quality of life is deteriorating day by day, care and safety about the weak person can't be guaranteed unless the problem is raised constantly through research[5].

To the next, with the advancement of ICT technology and the advancement of smart society and advanced society in the future, it is difficult for Shen Yun and Morality to develop together. In addition, the concept of distorted sexuality and hedonism have been influenced by children and adolescents, and they have become a subject of crime and sexual distortion. All children and adolescents must be protected in a safe and comfortable environment and have basic rights to grow. Nevertheless, crimes against children and adolescents are occurring. In modern society, the number of potential child offenders is increasing due to the dismantling of the family, the materialism, the pornography through the Internet, and the crime of the psychopath. In addition, children who are victims of the crime are increasingly exposed to crime in extreme situations such as intense competition in schools and family breakdowns, but the legal child protection system and social interest are low. Recently, as children's crime has increased and seriousness has become more serious, social interest has been increasing, but realistic alternatives have still been desperate. In fact, in the case of crimes against children, sexual violence, kidnapping, and abuse are organically linked to each other. Therefore, it is time to further develop and utilize technology to safely protect.

Finally, crimes against women classified as socially weak, along with the elderly, the disabled, and children, are increasing. In particular, women are exposed to various sex offenses according to changes in social sex culture. In addition, since most of the offenders are males, physical and physical countermeasures are disadvantageous and they are the target of crime. In recent years, the proportion of victims of violent crime has been increasing. Violent crime refers to murder, robbery, rape, forced harassment, theft, and violent incidents. On June 27, the National Statistical Office (NSO) and Ministry of Gender Equality and Family (MEXT) announced the "Life of Women in the 2013 Statistics". Eight out of ten victims of violent crime (murder, robbery, arson and rape) are women. In 2000, the number of victims totaled 6245 , or 71.3 percent of the total 8765 victims. In 2005, the number of victims increased to 79.9 percent of the total victims. In 2011, the number of victims of women totaled 20,354 , accounting for $83.8 \%$ of the total number of victims. As women are physically weak, you may think that they are not likely to become victims, but these figures are even higher than in other countries. According to data from the United Nations agency UNODC (United Nations Office on Drugs and Crime), when the victims of each country are classified by gender, the proportion of women victims of murder in 2008 is $51.0 \%$. It is very high compared with $22.5 \%$ of the US, $30.1 \%$ of China, $33.9 \%$ of Britain, $34.3 \%$ of France and $27.5 \%$ of Australia. Among the G20 countries, which are the main 20 countries, there is no country where the proportion of female victims is higher than Korea. Even in India, South Africa and Saudi Arabia, where women's rights are considered low, the proportion of women victims of murder does not exceed $30 \%[6]$.

Figure 1 shows violent crimes against men and violent crime against women. Since women have physical and physical weaknesses, violent crimes against women are constantly increasing.

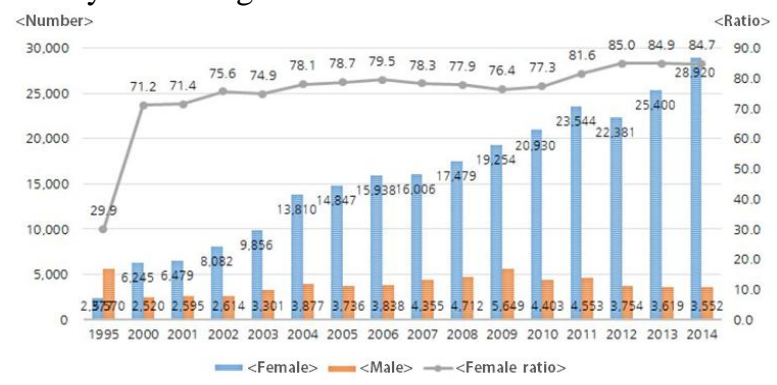

Fig. 1. Status of violent crime by sex, Korea

In this way, crimes against the socially vulnerable and the socially weak are continuously increasing, and it is urgent to take measures to prevent them. However, it is not possible to fundamentally reduce crimes against persons with disabilities as crime prevention measures such as installation of existing CCTV. A CCTV system can be of some help in solving problems after an accident, but it can never prevent accidents in advance[7]. 
The use of drones in the field of safety surveillance of vulnerable groups can provide a variety of benefits. First, there is a wider view on surveillance and inspection. It is possible to observe the entire area through the aerial photographing such that the visibility to the narrow area such as the sewer is secured not only in the air but also over the limit of the ground observation through the naked eye or CCTV. Second, accessibility is improved. High-rise buildings, alleys, and blind areas can be accessed easily by anyone who is approaching or difficult to identify with existing equipment. Third, illegal acts are more effective than CCTV. Compared to CCTV, which monitors only a given area in a fixed place, it can fly to a blind spot and monitor it. Fourth, it is easy to collect evidence. Expanded visibility, easy accessibility, and excellent imaging techniques make it easy to collect evidence[8]. The advantage of this kind of drone makes it possible to use it widely in the field of safety surveillance for vulnerable group.

\section{APPLICATION TECHNOLOGY}

\section{A. Principle of autonomous flight}

For the autonomous flight of a drone, a navigation system that can estimate its position is essential. A typical technique is SLAM (Simultaneous Localization and Mapping), which measures the relative distance to the surrounding environment by using a camera and a laser sensor to determine its own position. The self-localization technique creates a map of the surrounding environment and estimates its position to enable autonomous flight using it. These algorithms show accuracy of $\mathrm{cm}$ in the fixed environment and the low speed mobile environment.

There is a drone autonomous flight technology that enables self-location recognition in real time using UWB and autonomous communication using indoor wireless communication module. In addition, there is a stable drone autonomous flight technology that can recognize obstacles, analyze images, transmit them indoors using a wireless communication module, and utilize a controller with high computation speed. There is also an autonomous flight technique that measures the exact distance and position using self-position recognition and UWB using gradient descent algorithm. After all, the most important thing in the autonomous flight of the drone is to fly through communication with objects while recognizing and avoiding obstacles[9,10]. In other words, the key to avoiding obstacles in the drones' flight is the key. In the future, autonomous obstacle avoidance technology is essential for stable use of drones[11]. Figure 2 shows the concept of a drone-based safety accident prevention solution proposed in this paper.

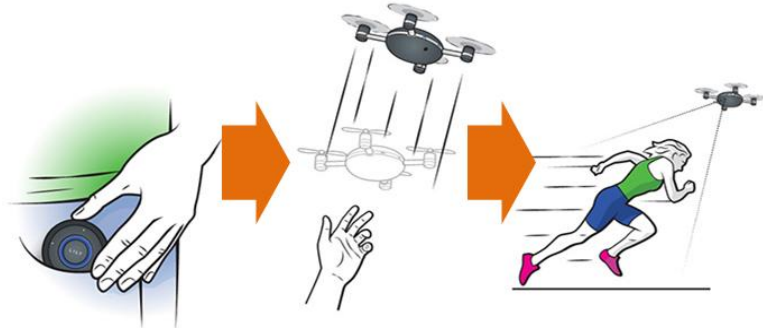

Fig. 2. Principle of autonomous drone of wireless communication

\section{B. Obstacle avoidance and object tracking}

Various algorithms have been applied to detect and avoid obstacles in drone flight. In recent years, fuzzy logic based algorithms, acoustic and optical information based algorithms, and rider sensor based algorithms have been utilized.

First, the fuzzy controller has the advantage of being able to cope effectively with nonlinear systems. Because drone is a nonlinear system, fuzzy logic based drone obstacle collision avoidance algorithm can cope effectively. The fuzzy logic based obstacle avoidance system can be constructed by merging two front ultrasonic sensors and one laser obstacle detection sensor and avoiding it by controlling the speed according to the obstacle distance. One distance measuring sensor is attached to the drone to control the altitude. Also, an object at a certain distance is measured through an ultrasonic sensor[12,13]. The maximum detection distance of the laser obstacle detection sensor can be detected from 3 5m, and the obstacle avoidance algorithm is composed of Safety, Normal, Hovering, and Braking. The safety stage does not detect any obstacles, so it will fly at a constant speed without reducing the speed. In the normal stage, left-right avoidance is performed based on fuzzy logic when an obstacle is detected In the hovering stage, if the left and right avoidance is not possible, the elevation rise will avoid the front obstacle. In the braking stage, it is judged that the distance between the obstacle and the obstacle is very close. As a result, the avoidance command for the obstacle is determined by the fuzzy logic $[14,15]$.

Next, there is an algorithm for avoiding obstacles based on data obtained by integrating acoustic information and optical information. That is, a method of setting a representative vector to an object and tracking it is applied to avoid an obstacle. Setting the representative vector on the object minimizes the movement of the dragon flight. In addition, the use of balloon-based airborne drone has advantages in terms of cost and size due to low energy consumption in maintenance of altitude. In addition, since the global information can be given to the object using the camera in the air, it is possible to reduce the risk of collision due to limitation of the beam width and detection distance when the drones are obstacle avoiding and performing tasks[16,17].

Finally, it is an obstacle avoidance algorithm using LIDAR technology. Light Detection and Ranging (LIDAR) is an active remote sensor system that samples laser pulses on a three-dimensional surface of an object and outputs point data (3D point cloud). These systems are widely used in various fields such as topographic mapping, filtering, power line and shoreline extraction based on the advantage of being able to quickly measure precise surface information of a three-dimensional object. In recent years, high speed lasers using $2 \mathrm{D}$ detectors such as video LIDAR have been developed and applied to fields requiring real-time processing such as flight obstacle detection and target tracking. In particular, the development and utilization of Geiger-mode imaging LIDAR sensors is increasing.

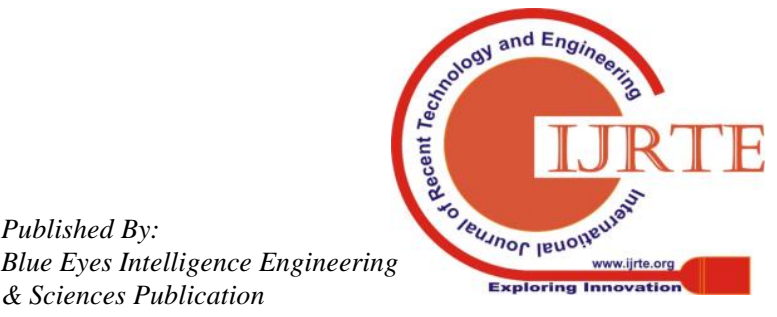




\section{Design of Real-time Safety Accident Prevention Solution for Socially Vulnerable using Object Recognition and Tracking Technology}

Through this, it is possible to model obstacle detection by linking the Geiger mode image LIDAR sensor with various high speed data analysis techniques, and avoid obstacles through simulation. Figure 3 shows the integrated use of a camera and a LIDAR sensor for collision avoidance and obstacle detection, which are essential for the autonomous flight of a drone.

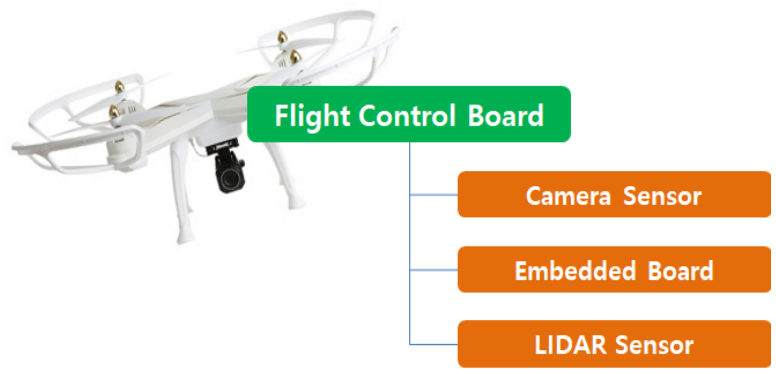

Fig. 3. LIDAR sensor-linked obstacle avoidance drone

\section{SYSTEM DESIGN}

We want a safe society, a society without crime, and a society that lives peacefully all over the world. In order to do this, various technologies, systems, and regulations as well as government policies should be integrated. As a result, it is absolutely necessary to develop and utilize ICT technology in order to pursue a safe society.

Therefore, in this paper, we have identified the problem of crime prevention system using existing CCTV system. To solve this problem, we have developed a safety accident prevention solution using drones. To this end, we designed the drones to enable real-time tracking by applying autonomous flight technology, obstacle avoidance technique, and object tracking technology. In addition, wireless communication between the object and the drone was applied to prevent flight deviation. And, it is designed to link emergency notification service through real-time video transmission during flight. Figure 4 is a conceptual diagram of the overall solution designed in this paper.

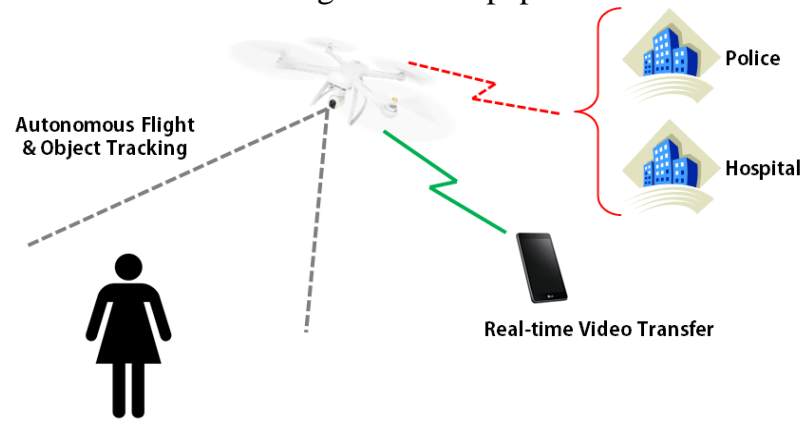

Fig. 4. Whole solution conceptual diagram

First, the obstacle avoidance technique was designed by integrating various techniques. Conventional ultrasonic obstacle avoidance technique is very difficult to avoid due to the short detection distance of the ultrasonic sensor. It is not suitable for industrial use because it can't be used for small-sized toys because it is impossible to perform precision detection. Also, the conventional camera-based obstacle avoidance technique has a problem that obstacle detection is limited due to only the view of the camera. In addition, the detection efficiency is very low for the achromatic object which is very sensitive to the light source and has no texture. Finally, the LIDAR sensor method has a disadvantage in that it can't obtain data when it touches a sparkling object such as a mirror. In addition, there is an inconvenience that measurement can be performed only when a special device such as a polarizing filter is used together with a specific object.

Therefore, it is designed to detect and avoid obstacles by integrating LIDAR sensor and camera method. That is, 3D mapping data is extracted by using 3D point cloud data obtained from LIDAR sensor and 2D image obtained from camera. As a result, SFM(Structure from Motion) matching is performed on the image captured by the camera during flight. At the same time, it detects and avoids obstacles by performing ICP(Iterative Closest Point) based matching of data scanned at each waypoint through the LIDAR sensor.

Figure 5 shows the SFM(Structure from Motion) matching for images taken by continuous shooting on a drone flight. Figure 6 shows the ICP(Iterative Closest Point) matching for the data scanned at each waypoint using the LIDAR sensor.

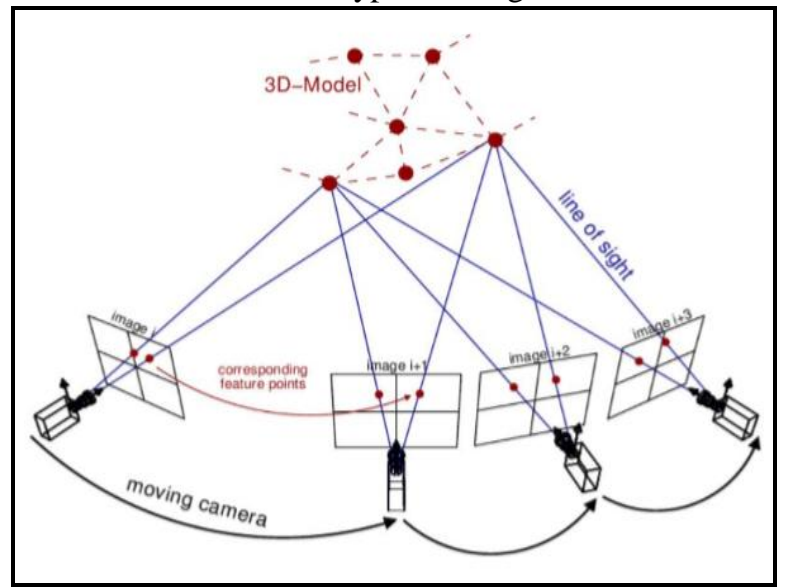

Fig. 5. Image SFM matching using camera

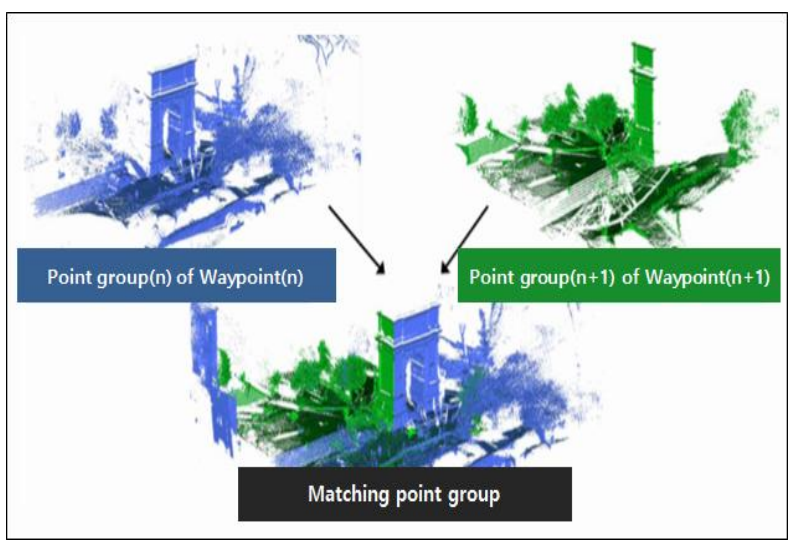

Fig. 6. Scan data ICP matching using LIDAR sensor

Second, algorithms are designed to calculate distance, flight speed, and collision time with obstacle. In other words, collision avoidance algorithm is designed by using collision expected time and relative velocity based on obstacle information. It can be divided into detection mode, tracking mode, and avoidance mode based on the estimated collision time and distance between the drones and obstacles.

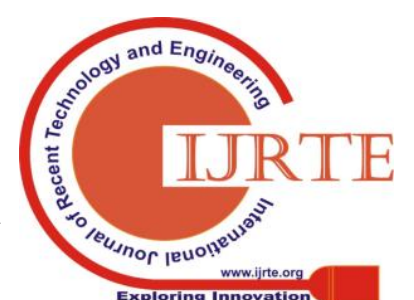


The detection mode is the navigation mode in which the drones fly toward the path point. While driving, use LIDAR to monitor the driving direction. Also, when an obstacle appears, the obstacle in the flight path is detected using the LIDAR sensor and the image sensor. If an obstacle is detected in the detection mode, it switches to the tracking mode. The tracking mode is a mode for acquiring the position information of the obstacle and recognizing the risk of collision with the drones. Using the LIDAR sensor and camera, calculate the expected collision time considering the distance between the drones and the obstacle, the flight speed and direction. Based on the estimated collision time with the obstacle and the relative distance, determines whether or not to switch to avoid mode and when to switch. The avoidance mode is a mode in which the position information of the obstacle calculated in the tracking mode and the safety radius that does not collide with the obstacle based on the speed of the drone are calculated. Modify the flight path by creating a new path point for the drone in the calculated safety radius range. Perform evasive maneuver through flight to new route. Figure 7 shows the algorithm for obstacle detection and collision avoidance. Each flight mode is determined according to the algorithm procedure and the drone flight proceeds.

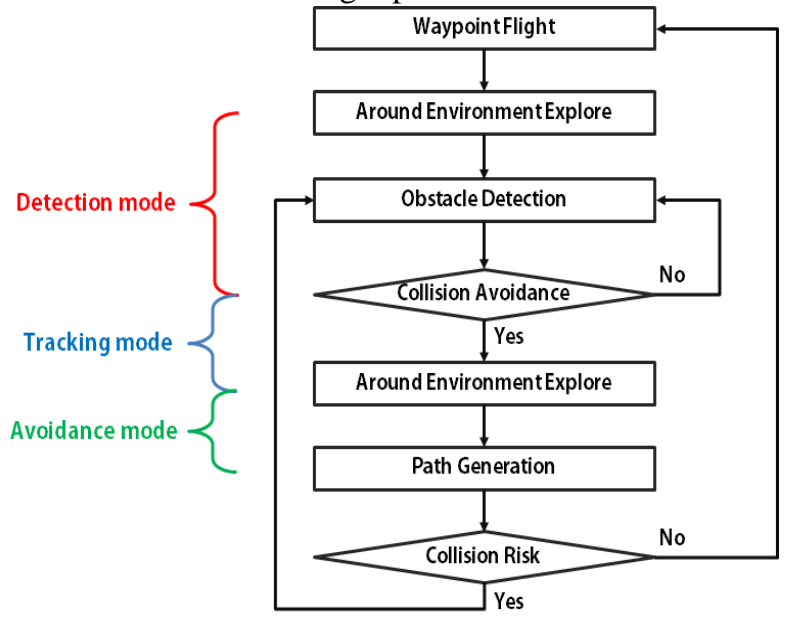

Fig. 7. Flight modes and collision avoidance algorithms

Finally, it is a design for real-time video transmission and location recognition module. To this end, drone are equipped with a mobile video transmitter and a position recognition device and obtain image information in real time. The obtained image information is made available through a commercial wireless communication network based on high speed packet access. In this manner, video and position sensor information transmitted in real time is displayed to the user.

In the video compression and real-time transmission module, the algorithm based on mobile high speed packet access is used. It is possible to simultaneously transmit additional data such as location information as well as real time video transmission through a network. In addition, the real-time image transmission module can be used in a high-speed packet access as well as a similar low-band wireless communication network such as GSM. In a relatively high-bandwidth wired network or short-range wireless LAN, higher-quality images can be transmitted more efficiently than existing products. Figure 8 shows the module design for real-time image transmission in wireless communication environment.

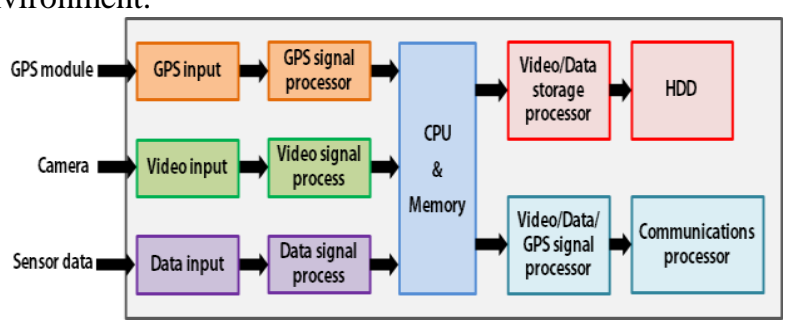

Fig. 8. Real-time video transfer module

\section{CONCLUSION}

In modern society, social safety problems are constantly occurring. In particular, crime targeting vulnerable groups is increasing. This is due to the fact that the physical and physical weaknesses of the victim are easy to use, making the safety problem more serious. CCTV is used to solve social safety problems and prevent crime. CCTV is deeply embedded in modern life and has become commonplace in both public and private places. Early CCTVs were first used by the US military for military purposes during the Vietnam War. Since then, it has become common use for general public safety purposes. In addition, the use of CCTV in the public sector by the police was mainly used for public purposes such as crime prevention, traffic information collection, and traffic control. In addition, the use of CCTV for safety is becoming common in private places such as apartments, apartment buildings, and elevators.

However, the CCTV surveillance system has the inconvenience that it has to be monitored by a staff of 24 hours in the central control and management room. In addition, there are problems such as additional installation of facilities and additional financial expenses such as additional installation of CCTV due to blind spot generation. As a result, although CCTV is widely used in various fields of society in general, there is a tendency to discuss the development and application of alternative technologies to complement the increase in efficiency and problems. There is a high possibility of infringing fundamental rights such as constitutional rights, privacy and freedom of privacy, and the right to self-determination, and a new approach to efficient installation, operation and management is needed.

Therefore, in this paper, we have identified the problem of crime prevention system using existing CCTV system. To solve this problem, we have developed a safety accident prevention solution using drones. To this end, we designed the drones to enable real-time tracking by applying autonomous flight technology, obstacle avoidance technique, and object tracking technology. In addition, wireless communication between the object and the drone was applied to prevent flight deviation. And, it is designed to link emergency notification service through real-time video transmission during flight. 


\section{REFERENCES}

1. N. J. Park, M. R. Kim, "Implementation of load management application system using smart grid privacy policy in energy management service environment," Clust. Comput., Vol. 17, No. 3, 2014, pp. 653-664.

2. M. Aguado, E. Jacob, J. Matias, C. Conde, Berbineau M, "Deploying CCTV as an Ethenet service over the WiMAX mobile network in the public transport scenario," Proceedings of IEEE International Conference on Communications Workshops, 2009.

3. Beyan, Cigdem, A. Temizel, "Adaptive mean-shift for automated multi object tracking," IET Comput. Vis., Vol. 6, No. 1, 2012, pp. 1-12.

4. Y. S. Park, "A Study on the Criminal Victim Compensation Program in Korea," Correctional welfare research, Vol. 25, 2012, pp. 117-141.

5. C. Harris, P. Jones, D. Hillier, D. Turner, "CCTV surveillance systems in town and city centre management," Property Management, Vol. 16, No. 3, 1998, pp. 160-165.

6. H. Kim, "Current Situation and Countermeasures of Purchasing Sex from Children or Juveniles," Korean Journal of Criminology, Vol. 29, No. 3, 2007, pp. 67-90.

7. S. Rusitschka, K. Eger, C. Gerdes, "Smart grid data cloud: A model for utilizing cloud computing in the smart grid domain," First IEEE International Conference on Smart Grid Communications (SmartGridComm), Vol. 1, No. 1, 2010, pp. 483-488.

8. Dario Floreano, J. Robert, "Wood. Science, technology and the future of small autonomous drones," Nature, Vol. 521, 2015, pp. 460-466.

9. H. Lim, J. M. Park, D. W. Lee, H. J. Kim, "Build your own quadrotor: Open-source projects on unmanned aerial vehicles," IEEE Robotics \& Automation Mag., Vol. 19, No. 3, 2012, pp. 33-45.

10. Vijay Kumar, Nathan Michael, "Opportunities and challenges with autonomous micro aerial vehicles," The International Journal of Robotics Research, Vol. 31, No. 11, 2012, pp. 1279-1291.

11. Y. Wang, e. K. Teoh, D. Shen, "Lane detection and tracking using B-Snake," Image and Vision Computing, Vol. 22, No. 4, 2004, pp. 269-280.

12. Kimberly McGuire, Guido de Croon, Christophe De Wagter, Karl Tuyls, Hilbert Kappen, "Efficient Optical Flow and Stereo Vision for Velocity Estimation and Obstacle Avoidance on an Autonomous Pocket Drone,' IEEE Robotics and Automation Letters, Vol. 2, No. 2, 2017, pp. 1070-1076.

13. H. Hirschmuller, "Stereo processing by semiglobal matching and mutual information,” IEEE Trans. Pattern Anal. Mach. Intell., Vol. 30, No. 2, 2008, pp. 328-341.

14. C. De Wagter, S. Tijmons, B. Remes, G. de Croon, “Autonomous flight of a 20-gram flapping wing MAV with a 4-gram onboard stereo vision system," Proc. IEEE Int. Conf. Robot. Autom., 2014, pp. 4982-4987.

15. S. Tijmons, G. de Croon, B. Remes, C. De Wagter, M. Mulder, "Obstacle avoidance strategy using onboard stereo vision on a flapping wing MAV," arXiv:1604.00833, 2016.

16. Peng Yao, Honglun Wang, Zikang Su, "Cooperative path planning with applications to target tracking and obstacle avoidance for multi-UAVs," Aerospace Science and Technology, Vol. 54, 2016, pp. 10-22.

17. Peng Yao, Honglun Wang, Zikang Su, "Real-time path planning of unmanned aerial vehicle for target tracking and obstacle avoidance in complex dynamic environment," Aerospace Science and Technology, Vol. 47, 2015, pp. 269-279.

\section{AUTHORS PROFILE}

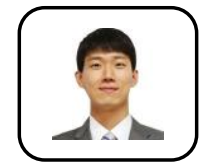

Sung-Jin Jeoung Received the M.S. degrees from the Seoul Business School MBA of Seoul School of Integrated Science \& Technologies. He is currently a Ph.D. candidate in the Seoul Business School of aSSIST. and IT management His research interests include Drone, IoT applications,

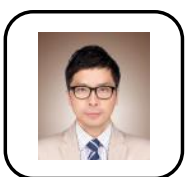

Bong-Hyun Kim Received the Ph.D. degrees from the Department of Computer Engineering of Hanbat National University. He is currently a professor in the Department of Smart IT, U1 University, Korea. His research interests include Data analysis, IoT, USN applications, and Healthcare system. 